Manuel Ceccarelli

\title{
Milchzauber und Verwandtes in sumerischen Texten
}

https://doi.org/10.1515/fabula-2017-0032

\begin{abstract}
The widespread belief in the existence of witches who can steal cow milk can also be traced in the ancient Sumerian literary text Enmerkara und Ensukukešdana. A sorcerer bewitches the cows and the goats of a goddess so that they cannot give milk and cream anymore: the cream moves up to the horns and the milk retreats to the back of the animals. The story also shows an interesting similarity with some European traditions according to which dry cows have milk in their horns. Moreover, we find in some Mesopotamian incantations the belief that the evil eye and demons can mar milk and cheese, a belief that survives to this day in the folklore of many European countries, Syria and Iraq.
\end{abstract}

Zusammenfassung: Der weit verbreitete Glaube an Milchhexen findet eine Parallele in dem sumerischen literarischen Text Enmerkara und Ensukukešdana. Dort werden die Kühe und Ziegen einer Göttin von einem Geisterbeschwörer verhext, so dass sie keine Milch mehr geben können: Der Rahm soll sich in die Hörner, die Milch in den Rücken zurückziehen. Die Erzählung bezeugt eine interessante Ähnlichkeit mit einigen Aspekten europäischen Volksglaubens, denen zufolge die trockene Kuh ihre Milch in den Hörnern hat. Wiein Europa, in Syrien und im Irak lässt sich auch in einigen sumerischen Beschwörungen die Vorstellung belegen, dass sowohl das böse Auge als auch Dämonen Milch und Käse verderben können.

\section{Einführung}

In verschiedenen Volkstraditionen trifft man auf die Vorstellung, dass Hexen und Dämonen die Kuhmilch stehlen beziehungsweise verderben können. Auch dem bösen Blick wird eine verheerende Wirkung auf die Genießbarkeit der Milch und die Milchergiebigkeit der Kühe zugeschrieben. Die weite Verbreitung dieser Vorstellungen entnimmt man bereits dem Handwörterbuch des deutschen Aberglaubens. Dort werden dem Eintrag ,Milchhexe“ dreißig Seiten gewidmet, die eine Fülle an Hinweisen über Milch raubende Hexen und Geister in mittel- und

Manuel Ceccarelli, Université de Genève, Sciences de l'antiquité; Bern, Schweiz, E-Mail: manuel.ceccarelli@unige.ch 
osteuropäischen Traditionen bieten. ${ }^{1}$ Auch Stith Thompson verzeichnete in seinem Motif-Index verschiedene Motive, die auf den erwähnten Volksglauben zurückgehen. ${ }^{2}$ Diese Motive und Vorstellungen lassen sich ebenfalls in einigen Texten aus dem alten Mesopotamien belegen, die anscheinend bis heute noch nicht in einen Zusammenhang mit dem verbreiteten Volksglauben an die Existenz von Milchhexen und Milchzauber gebracht worden sind.

\section{Milchzauber}

In der sumerischen Komposition Enmerkara und Ensukukešdana erscheint das Motiv der Verhexung von Kühen und Ziegen, deren Euter ein Zauberer austrocknen lässt. Bei diesem Literaturwerk handelt es sich um ein Streitgedicht über zwei legendäre Herrscher: Enmerkara, Herrscher der südmesopotamischen Stadt Uruk, und Ensukukešdana, Herrscher der legendären Stadt Arata, die den Epen nach östlich von Mesopotamien lag. Ensukukešdana verlangt die Unterwerfung von Enmerkara und mit der Absicht den Gegner zu besiegen, bedient er sich eines Geisterbeschwörers, Uralimnuna. Dieser begibt sich nach Ereš, der Stadt Nisabas, der Göttin des Getreides und der Schreibkunst, die offenbar im Einflussbereich der Stadt Uruk lag. Dort verhext er die Kühe und die Ziegen der Göttin, damit diese keine Milch mehr geben und die Versorgung der Stadt Uruk eingestellt werden muss. Es kommt dann zu einem magischen Wettstreit zwischen Uralimnuna und einer alten Frau Namens Saĝburu. ${ }^{3}$ Die alte Frau besiegt den Geisterbeschwörer und verurteilt ihn zum Tode. Ensukukešdana erkennt schließlich Enmerkaras Überlegenheit an. Die für unser Thema relevanten Zeilen lauten in Übersetzung: ${ }^{4}$

171. Er (d. h. der Geisterbeschwörer) näherte sich dem ,Haus der Hürde‘, dem Haus, in dem die Kühe leben.

172. Die Kühe schüttelten in der Hürde den Kopf.

173. Er lässt die Kuh sprechen. Diese unterhält sich mit ihm wie ein Mensch:

1 Eckstein 1934-1935, 293-352.

2 Mot. D 2083: Evil magic in the dairy; F 451.3.3.5: Dwarfs bewitch cows to give no milk; $M$ 471.1: Curse: cow will give red milk.

3 Auch diese Passage ist aus der Perspektive der Märchenforschung interessant. Fünfmal fischt der Geisterbeschwörer jeweils ein beziehungsweise zwei Tiere aus einem Fluss, doch jedes Mal fischt die alte Frau ein großes Raubtier, das die andere Tiere ergreift und fortbringt. Selbst die Figur der ,alten Frau', hier wohl in der Rolle einer ,weisen Alten' mit magischen Fähigkeiten, ist in dieser Hinsicht interessant. Zum Motiv der ,alten weisen Frau‘ vgl. z. B. Riedel 1995; Gobrecht 2000, 131-137; dies. 2010, 128-131. Eine Bearbeitung dieser Stelle unter Berücksichtigung der Figur der ,weisen Alten' durch den Verfasser befindet sich in Vorbereitung.

4 Die Zeilen wiederholen sich für die Ziege verbatim und werden deswegen hier ausgelassen. 
174. „Kuh, wer isst deinen Rahm? Wer trinkt deine Milch?“

175. „Meinen Rahm isst Nisaba,

176. meine Milch trinkt Nisaba.

177. Mein Käse, eine makellose Krone, die kunstvoll hergestellt ist,

178. wird dem großen Speisesaal, Nisabas Speisesaal, zur Zierde gereichen.

179. Solange ich meinen Rahm aus der reinen Hürde liefere,

180. solange ich meine Milch aus der reinen Hürde liefere,

181. wird keiner die echte Wildkuh Nisaba, die Erstgeborene Ellils,... aufstehen lassen.“

182. „Kuh, dein Rahm zu deinem Horn! Deine Milch zu deinem Rücken!“

183. Und die Kuh: ihr Rahm wurde zu ihrem Horn (zurück)gezogen! Ihre Milch wurde zu ihrem Rücken (zurück)gezogen!

197. Damals wurde in den Hürden und in den Pferchen ein ,stilles Haus' ausgerufen; die Verwüstung wurde angerichtet:

198. In den Eutern der Kühe gab es keine Milch (mehr), Dunkelheit breitete sich über das Kalb aus. ${ }^{5}$

199. Das junge Kalb hatte Hunger und weinte bittere Tränen. ${ }^{6}$

Diese Passage entspricht genau Mot. D 2083.1: Cows magically made dry. Besonders interessant ist die Aussage, dass sich der Rahm in die Hörner zurückziehen soll. Die Vorstellung, dass die Milch der trockenen Kühe in den Hörnern steckt, war in Mitteleuropa bekannt und prägte auch einige Redewendungen und Sprichwörter. Im Wörterbuch der westfälischen Mundart liest man: „In der Mark (auch zu Rheda) sagt man von der güsten Kuh, sie habe die Milch in den Hörnern.“7 Man vergleiche hierzu auch das alte deutsche Sprichwort: „Die Milch

5 Wörtlich: „In den Eutern der Kühe gab es keine Milch (mehr), dies ließ die Sonne über dem Kalb heruntergehen.“

6 Vgl. Wilcke 2012, 62-64, 82 f., und Attinger 2015:

171. $\mathrm{e}_{2}$-t $\left[\mathrm{ur}_{3}\right]-\mathrm{ra} \mathrm{e}_{2} \mathrm{ab}_{2}$ dur $_{2}$-ru-na-aš ba-te

172. $\mathrm{ab}_{2}$-[e tu $] \mathrm{r}_{3}$-ra saĝ mu-da-ab-sag

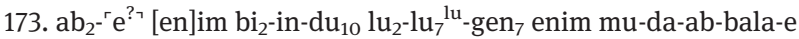

174. $\mathrm{ab}_{2} \mathrm{i}_{3}$-zu a-ba-a $\mathrm{i}_{3}$-gu - -e ga-zu a-ba-a $\mathrm{i}_{3}-\mathrm{na}_{8}-\mathrm{na}_{8}$

175. $\mathrm{i}_{3}-\hat{g}_{10} \mathrm{i}_{3}-\mathrm{gu}_{7}-\mathrm{e}{ }^{\mathrm{d}}$ nisaba-ke ${ }_{4}$

176. ga-ĝ $\mathrm{gu}_{10} \mathrm{i}_{3}-\mathrm{na}_{8}-\mathrm{na}_{8}{ }^{\mathrm{d}}$ nisaba-ke ${ }_{4}$

177. [ga-ara - - $\mathrm{g}$ ] $\mathrm{u}_{10} \mathrm{suku}_{6} \mathrm{ku}_{3}$ galam du $\mathrm{u}_{11}$-ga

178. [ $\mathrm{unu}_{2}$ gal] $\mathrm{unu}_{2}{ }^{\mathrm{d}}$ nisaba-še $\mathrm{e}_{3}$ me-te-a-aš im-mi-ib-ĝal ${ }_{2}$

179. $\mathrm{i}_{3}$ - $\mathrm{gu}_{10}[\mathrm{tu}] \mathrm{r}_{3}{ }^{\mathrm{K}} \mathrm{ku}_{3}{ }^{7}$-ta en-na(-)ga-mu-DU-a-aš

180. ga-ĝu $u_{10}$ tur $_{3} \mathrm{ku}_{3}$-ta e[n-n]a(-)ga-mu-DU-a-aš

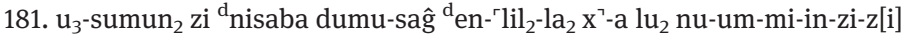

182. $\mathrm{ab}_{2} \mathrm{i}_{3}$-zu si-muš $\check{3}_{3}-z u-\check{c}_{3}$ ga-zu murgu $\mathrm{u}_{2}-\mathrm{z}\left[\mathrm{u}-\check{s ̌}_{3}\right]$

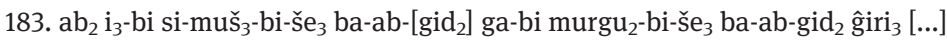

197. $\mathrm{u}_{4}$-bi-a tur ${ }_{3}$ amaš-a e e-si-ga ba-ab-du en $_{11}$ ni $\hat{g}_{2}$ ha-lam-ma ba-ab-AK

198. akan ab ${ }_{2}$-ka ga nu-un-ĝal ${ }_{2}$ amar-e $\mathrm{u}_{4} \mathrm{bi}_{2}-\mathrm{ib}_{2}{ }^{\text {?? }}$-šuš 2

199. [a]mar tur-bi ša ${ }_{3}$ su $_{3}$-ga mu-un- ${ }^{\top} \hat{g a l}_{2}{ }^{\urcorner} \mathrm{ir}_{2}{ }^{\ulcorner} \mathrm{ge}_{17}{ }^{\top} \mathrm{i}_{3}-\mathrm{še}_{22}-\check{s}\left[\mathrm{e}_{22}\right]$.

7 Woeste 1882, 94. 
ist der Kuh in die Hörner gegangen. “8 Dieselbe Vorstellung findet man in einer litauische Redensart wieder: „Die Milch sammelt sich in die Hörner ${ }^{\text {[sic!] }}$ (d. i. die Kuh hört auf Milch zu geben). “9 Dieser Glaube lässt sich ebenfalls aus manchen mitteleuropäischen Zauberpraktiken erschließen.

Paul Sebillot berichtet in seinem Le folk-lore de France von einem Zauberritus der Bauern von Coiron (Ardèche): Man stellte zwei gekreuzte Ginsterzweige zwischen die Hörner der Kuh und legte zwei Buchsbaumzweige auf ihren Schwanz. Dabei sagte man: „Crous de Saint André duono dé lè, crous de Barabas n'en douno pas.“ Dann wurden der Kuh einige Schwanzhaare ausgerissen und verbrannt, sobald diese ein Kalb geboren hatte. Bei den Bauern von Lüttich, wurde eine Zauberpraktik an den Hörnern und am Schwanz der trockenen Kuh vollzogen, die die trockene Kuh wieder ergiebig machen sollte. Bei der letzten Handlung des Ritus musste man das rechte Horn der Kuh mit der linken Hand anfassen und dabei „Merci, ma vache“ sagen; dann konnte die Kuh wieder reichlich Milch geben. ${ }^{10}$ Bezüglich der Zauberpraktiken der belgischen Bauern äußerte sich bereits Wilhelm Mannhardt in seinem Buch Germanische Mythen folgendermaßen: „Diese Sitte bezieht sich auf den Aberglauben, dass die trockenen Kühe die Milch in den Hörnern haben“. ${ }^{11}$ Wenn in Ostpreußen eine Hexe die Milch einer Kuh nahm, musste man die Hörner des Tieres mit Knoblauch reiben, ein Säckchen mit magischen Gegenständen an den Schwanz binden und eine Zauberformel aussprechen. ${ }^{12}$ Wenn man im oberen Nahetal eine frische Kuh kaufte, so musste man den Strick, an dem man sie nach Hause geführt hatte, um die Hörner schlingen und dort drei Tage lassen, damit sich die Milch nicht verziehe. ${ }^{13}$

Möglicherweise hing auch die Praxis der Enthornung der Milchkühe mit dem geschilderten Glauben zusammen. Man dachte nämlich, dass Kühe mit kleinen beziehungsweise abgesägten Hörnern mehr Milch als solche mit langen Hörnern geben würden. ${ }^{14}$ Die Enthornung könnte ursprünglich eine Maßnahme zur Hemmung der Wanderung der Milch in die Hörner gewesen sein.

\footnotetext{
8 Wander 1873, 659 (Nr. 51).

9 Schleicher 1857, 172.

10 Sebillot 1906, 85.

11 Mannhardt 1858, 35, Anm. 4.

12 Seligmann 1910, 286.

13 Wolff 1905, 293.

14 Ganot 1862. Ganot erklärt die Enthornung als eine wirtschaftlich sinnvolle Praxis, denn sonst würden die Hörner Nahrungsstoffe verbrauchen, die der Milchproduktion zugute kommen könnten. Es gab allerdings auch Gegenstimmen: In der Neuen Bürger- und Bauern-Zeitung 10 (1843) 367, liest man, es gebe keinerlei Verbindungen zwischen Hörnern und Milchproduktion. Der Glaube, die Milchergiebigkeit hänge mit der Länge der Hörner zusammen, sei im Analogie-
} 


\section{Verderben von Milch und Käse als Folge des bösen Blicks}

Auch der böse Blick kann die Euter der Kühe austrocknen beziehungsweise die Qualität der Milch so beeinträchtigen, dass sie nicht mehr genießbar ist. ${ }^{15}$ Der Glaube an den bösen Blick war selbst im alten Mesopotamien sehr verbreitet und dementsprechend wurden spezielle Beschwörungen vorgenommen, um ihn abzuwehren. ${ }^{16}$ Unter den Wirkungen des bösen Blickes zählt auch das Verderben (wörtlich „Vernichten“) des Käses:

1. Das Auge: ein Drache. Das Auge des Menschen: ein Drache.

2. Das Auge des böswilligen Menschen: ein Drache.

3. Es hat sich dem Himmel genähert und (jetzt) regnet es nicht mehr.

4. Es hat sich der Erde genähert und (jetzt) wachsen Kräuter und Gras nicht mehr.

5. Es hat sich den Ochsen genähert und hat deren Joch gelöst.

6. Es hat sich dem ,Haus der Hürde‘ genähert und hat dessen Käse gänzlich vernichtet. ${ }^{17}$

Im heutigen Syrien und Irak wird nicht nur dem bösen Blick eine verheerende Wirkung zugeschrieben, sondern auch Tabubrüche sowie die Unreinheit setzen die Menschen, insbesondere schwangere und stillende Frauen sowie Babys, dem Einfluss des kebsa aus. Es handelt sich dabei um eine schädigende Kraft, die solche Missstände bestraft. ${ }^{18} \mathrm{Zu}$ seinen Auswirkungen zählt das Austrocknen der Muttermilch bei stillenden Frauen. ${ }^{19}$

schluss dadurch entstanden, dass die milchergiebigen holländischen Kühe kurze und krumme Hörner besitzen. Die Enthornung wird heute in der Massentierhaltung durchgeführt, um Verletzungen von Tieren und Menschen zu vermeiden.

15 Eckstein 1934-35, 314.

16 Zum bösen Blick in Mesopotamien vgl. Geller 2003; Thomsen 1992; Schwemer 2007, 68.

17 Vgl. Geller 2003, 129-134, und Thomsen 1992, 31 f.:

1. igi-muš-huš igi lu $\mathrm{l}_{2}$-[ $\left[u_{7}\right]$ muš-ḩuš

2. igi lu $u_{2}$ ni $\hat{g}_{2}$-ḩulu $\operatorname{dim}_{2}$-ma muš-huš

3. an-ne ${ }_{2}$ ba-te im nu-še $\hat{g}_{3}$-še $\hat{g}_{3}$

4. ki-a ba-te $u_{2} \check{s}$ em nu-mu $-\mathrm{mu}_{2}$

5. gud-e ba-te ${ }^{\text {seššutul }}$

6. e e $_{2}$-tur-e ba-te ga-ra-bi im-ta-gaz-gaz.

18 Drower 1938; Fartacek 2010, 98-102.

19 Drower 1938, $105 \mathrm{f}$. 


\section{Raub und Verderben der Milch durch Dämonen}

Nicht nur Hexen, sondern auch Dämonen, Kobolde ${ }^{20}$, der Teufel, ${ }^{21}$ Mahre, Totengeister, Drachen und andere Wesen ${ }^{22}$ können die Milch stehlen. Ebenfalls lässt sich in Mesopotamien der Glaube belegen, dass Dämonen Milch und Rahm verderben können. Man vergleiche hierzu folgende Beschwörung:

29. Er brach? die Erde durch: Der Zahn ist der Zahn eines Krokodils.

30. Der Eckzahn... ist der Eckzahn eines Welpen.

31. Bei der Rahmträgerin (/dem Rahmträger) vernichtet er den Rahm,

32. Bei der Milchträgerin (/dem Milchträger) vernichtet er die Milch.

33. Derjenige, der die Ammen beißt, die ihren starken Griff

34. auf ihren großen (Kindern)

35. (und) auf ihren kleinen (Kindern) gelöst haben - oh Asalluhi ${ }^{23}-$,

36. er greift selbst mich an!

37. Der Asag-Dämon ${ }^{24}$ hat sich dem Jungen genähert: Weiche von hier!

38. Wortlaut der Beschwörung: Es ist (eine Beschwörung) gegen den angreifenden UdugDämon. $^{25}$

Noch im heutigen Syrien trifft man auf den Glauben, dass Dämonen die Euter der Kühe austrocknen können. Gebhard Fartacek referiert eine einschlägige Erzählung aus dem Ğebel az-Zāwiya (Nord-West Syrien): Ein Ǧinn ließ die Kuh eines

20 Eckstein 1934-35, 295 f.

21 Eckstein 1934-35, 299.

22 Eckstein 1934-35, $320 \mathrm{f}$.

23 Der Gott Asalluhi galt als Sohn des Beschwörungsgottes Enki und spielte als Helfer der Erkrankten eine wichtige Rolle in den Beschwörungen.

24 Asag und Udug sind Dämonen, die die Menschen heimsuchen können, vgl. Steinert 2012, 303-314.

25 Vgl. van Dijk 1985, 49 (Nr. 86):

29. ki in-dar? $\mathrm{zu}_{2} \mathrm{zu}_{2}$ kušu ${ }^{\mathrm{ku}}{ }^{\mathrm{k}}$-kam

30. gug $_{6}$ ? BA gug 6 ur tur-ra-kam

31. $\mathrm{i}_{3}$ guru $_{6} \mathrm{i}_{3}$ mu-un-da-gaz-e

32. ga guru 6 ga mu-un-da-gaz-e

33. eme $_{2}$-da lirum kala-ga-bi

34. gal-gal-bi tuh-a

35. $\mathrm{zu}_{2}$ mu-un-ku${ }_{5}$-da TUR.TUR-bi tuh ${ }^{\mathrm{du}}$-a / asal-lu ${ }_{2}$-hi

36. $\mathrm{ni}_{2}-\hat{\mathrm{g}} \mathrm{u}_{10}$ mu-un-du $-d u_{7}$

37. $\mathrm{a}_{2}$ - $\mathrm{sag}_{3}$ guruš ba-te gaba-zu zi-ba-ra-ab

38. ka-enim-ma 'udug ${ }^{\top} \mathrm{du}_{8}$-du $\mathrm{du}_{8}$-da-kam.

Ich danke Dr. Enrique Jiménez für die Zusendung einiger Fotos der Tafel. 
Bauers austrocknen, der ein inzestuöses Verhältnis mit seiner Tochter hatte. ${ }^{26}$ Dadurch hatte der Ǧinn den Tabubruch bestraft. ${ }^{27}$

\section{Schlussbetrachtungen}

Die mesopotamische Literatur ist außerordentlich reich an verschiedenen Textgattungen und Traditionen. Dabei sind die besprochenen Texte ein gutes Beispiel dafür, wie ein Volksglaube in verschiedenen Textgenres Eingang fand. In dem vorliegenden Fall handelt es sich um den Glauben, dass Milch und Kühe gefährlichen magischen und dämonischen Einflüssen ausgesetzt sind. Wir befinden uns in der glücklichen Lage, sowohl die literarische Formulierung in Enmerkara und Ensukukešdana als auch die für den praktischen Gebrauch bestimmten Beschwörungen nachlesen zu können. Diese und der kulturanthropologische Vergleich mit den mitteleuropäischen und syrischen Traditionen verleihen der zitierten Stelle aus Enmerkara und Ensukukešdana, die sich somit nicht als bloße literarische Erfindung erweist, eine besondere Tiefe und lassen sie als Ausdruck realer, alltäglicher Vorstellungen und Ängste der (mesopotamischen) Menschen erscheinen.

\section{Literatur}

Attinger, Pascal: Enmerkara und Ensukukešdana, http://www.iaw.unibe.ch/unibe/portal/ fak_historisch/dga/iaw/content/e39448/e99428/e122665/e122821/pane122850/ e122904/1_8_2_4_ger.pdf. 2015.

van Dijk, Johannes Jacobus Adrianus u. a.: Early Mesopotamian Incantations and Rituals. New Haven 1985.

Drower, Ethel Stefana: Woman and Taboo in Iraq. In: Iraq 5 (1938) 105-117.

Eckstein, Franz: Milchhexe. In: Handwörterbuch des deutschen Aberglaubens 6. Hgg. Hans Bächtold-Stäubli/Eduard Hoffamnn-Krayer. Berlin 1934-1935, 293-352.

El-Shamy, Hasan: Folktales of Egypt. Collected, Translated, and Edited, with Middle Eastern and African Parallels. Chicago/London 1980.

El-Shamy, Hasan: Folk Traditions of the Arab World. A Guide to Motif Classification. Bloomington 1995.

26 Fartacek 2010, $119 \mathrm{f}$.

27 Die Bestrafung eines illegitimen sexuellen Verhältnisses durch das Austrocknen der Euter der Kuh lässt sich wohl als Variante von Mot. Q 552.3.03: Failure of crops and milk as punishment for adultery verstehen. Dieses Motiv ist auch in Ägypten belegt, s. El-Shamy 1980, 275 f.; ders. 1995/1, 327 zu Mot. Q 552.3.05\$. 
Fartacek, Gebhard: Unheil durch Dämonen? Geschichten und Diskurse über das Wirken der Ǧinn. Eine sozialanthropologische Spurensuche in Syrien. Wien/Köln/Weimar 2010.

Ganot, Eugen: Über ungehörnte Rindviehracen. In: Landwirtschaftliches Centralblatt für Deutschland. Repertorium der wissenschaftlichen Forschungen und praktischen Erfahrungen im Gebiete der Landwirtschaft 10 (1862) 330-332.

Geller, Markham J.: Paranoia, the Evil Eye, and the Face of Evil. In: Literatur, Politik und Recht in Mesopotamien. Festschrift für Claus Wilcke. Hgg. Walter Sallaberger u. a. Wiesbaden 2003, 115-134.

Gobrecht, Barbara: Alt und/oder weise? Hexen im Märchen. In: Alter und Weisheit im Märchen. Forschungsberichte aus der Welt der Märchen. Hgg. Ursula Heindrichs/Heinz-Albert Heindrichs. München 2000, 123-140.

Gobrecht, Barbara: Hexen, Zauberinnen und weise Frauen in europäischen Zaubermärchen. In: Abenteuer am Abgrund - Außenseiter im Märchen. Hgg. Harlinda Lox/Renate Vogt. Königsfurt 2010, 116-135.

Mannhardt, Wilhelm: Germanische Mythen. Forschungen. Berlin 1858.

Riedel, Ingrid: Die weise Frau in uralt-neuen Erfahrungen. Solothurn/Düsseldorf ${ }^{4} 1995$.

Schleicher, August: Litauische Märchen, Sprichworte, Rätsel und Lieder. Weimer 1857.

Schwemer, Daniel: Abwehrzauber und Behexung Studien zum Schadenzauberglauben im alten Mesopotamien. Wiesbaden 2007.

Sebillot, Paul: Le folk-lore de France. Bd. 3: La faune et la flore. Paris 1906.

Seligmann, Siegfried: Der böse Blick und Verwandtes. Ein Beitrag zur Geschichte des Aberglaubens aller Zeiten und Völker. Bd. 1. Berlin 1910.

Steinert, Ulrike: Aspekte des Menschseins im Alten Mesopotamien. Eine Studie zu Person und Identität im 2. und 1. Jt. v. Chr. Leiden/Boston 2012.

Thompson, Stith: Motif-Index of Folk-Literature. A classification of Narrative Elements in Folktales, Ballads, Myths, Fables, Medieval Romances, Exempla, Fabliaux, Jest-Books and Local Legends. Enlarged Edition. Bloomington 1966.

Thomsen, Marie-Luise, The Evil-Eye in Mesopotamia. In: Journal of Ancient Near Eastern Studies 51 (1992) 19-32.

Wander, Karl Friedrich Wilhelm: Deutsches Sprichwörter-Lexikon. Bd. 3. Leipzig 1873.

Wilcke, Claus: The Sumerian Poem Enmerkar and En-Suhkes-Ana: Epic, Play, Or? Stage Craft at the Turn from the Third to the Second Millennium BC with a Score-Edition and a Translation of the Text. New Haven 2012.

Woeste, Franz: Wörterbuch der westfälischen Mundart. Norden/Leipzig 1882.

Wolff, Theodor: Volksglauben und Volksgebräuche an der oberen Nahe. Fortsetzung. In: Zeitschrift des Vereins für rheinische und westfälische Volkskunde 2 (1905) 277-309. 